\title{
Role of polymeric materials in preventing COVID-19 infection
}

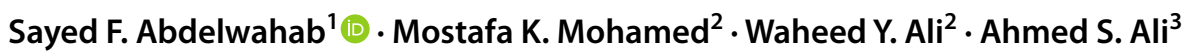

Received: 22 January 2021 / Accepted: 6 May 2021 / Published online: 6 July 2021

(C) The Author(s), under exclusive licence to Springer-Verlag GmbH Austria, part of Springer Nature 2021

\begin{abstract}
The safety of personal protective equipment (PPE) is very important, and so is the choice of materials used. The ability of electrostatic charges (ESCs) generated from the friction of engineered materials to attract or repel viruses has a significant impact on their applications. This study examined the ESCs generated on the surface of PPE used by healthcare workers to enhance their potential effectiveness in protecting the wearer from viruses. This is a crucial consideration for the newly emerged severe acute respiratory syndrome corona virus 2 (SARS-CoV-2), which has a negative charge. The magnitudes and signs of generated ESCs on the surfaces of the PPE were determined experimentally using an Ultra Stable Surface DC Voltmeter. The high negative ESCs acquired by the polyethylene disposable cap and facemask are expected to repel negatively charged viruses and prevent them from adhering to the outer layer of the material. Also, the choice of polypropylene for facemasks and gowns is excellent because it is an aggressively negatively charged material in the triboelectric series. This property guarantees that facemasks and gowns can repel viruses from the wearer. However, the positive ESCs generated on latex glove surfaces are of great concern because they can attract negatively charged viruses and create a source of infection. In conclusion, it is necessary to ensure that PPE be made of materials whose surfaces develop a negative ESC to repel viruses, as well as to select polyethylene gloves.
\end{abstract}

\section{Introduction}

Electrostatic charges (ESCs) generated from the friction of engineered materials have important implications for their applications as the use of polymeric materials increases. ESC built up on human skin and/or clothes in direct contact with the human body has the potential to attract or repel viruses, with potential implications for human health and safety. Viral particles may have a net charge, depending on the cumulative charges of their genetic material and that of the surface proteins. The genetic material (DNA or RNA) $[17,23]$ provides a partial negative charge, and indeed most

Handling Editor: Tim Skern.

Sayed F. Abdelwahab

icpminia@yahoo.com; sayed.awahab@mu.edu.eg

1 Department of Pharmaceutics and Industrial Pharmacy, Taif College of Pharmacy, Taif University, Al-Haweiah, P.O.

Box 11099, Taif 21944, Kingdom of Saudi Arabia

2 Department of Production Engineering and Mechanical Design, Faculty of Engineering, Minia University, Minia 61511, Egypt

3 Department of Mechanical Engineering, Faculty of Engineering, Suez Canal University, Suez 41522, Egypt viruses are reported to have a negative ESC [11, 12]. One study suggested that a residual net negative charge exists in the core of coronaviruses [20], while the crystal structure of the N-terminal domain (N-NTD; lumen) of the N protein of severe acute respiratory syndrome coronavirus 2 (SARS$\mathrm{CoV}-2$ ) exhibits localized segregation of positive and negative charges [13].

The natural charge of viruses can be exploited for several purposes. An applied electric field can be used for capture or repulsion of viral particles [14]. For example, protective electrostatic composite fiber fabrics made of a graphene oxide (GO) layer mixed with polymethyl methacrylate (PMMA) was designed to repel the negatively charged spike proteins of airborne viruses [23]. In addition, the strong electric field alters the induced dipole of the viral proteins [19], causing permanent damage in the form of electroporation, which allows positive salt ions to diffuse into the virus, leading to its inactivation and disintegration. Importantly, a few recent studies used this principle and an electric field over metallic foam to disinfect water [18, 21]. The process depends on a coating of carbon nanotubes (CNT) over the metallic filter where electroporation is carried out to disable the viruses. Similarly, an X-ray-enhanced corona system has been shown to be effective for nanoparticle capture and 
could be used not only to capture viral particles [10] but also to completely inactivate the trapped biological particles.

Building on the above, experiments were carried out recently to develop clothing that could inactivate or repel coronaviruses such as that causing coronavirus disease 2019 (COVID-19) [6]. This approach depends on electroceutical materials that generate electric fields across the fabric surface to disrupt the behavior of bacteria or viruses. To achieve such an effect, spots of silver and zinc, one to two millimeters wide and spaced one millimeter apart, were printed on polyester textile. The virus that causes COVID19, SARS-CoV-2, is known to have a negative ESC [15] and, according to initial investigations, to spread via droplets and the airborne route $[9,22,24]$. In line with these traits, when the designed textile is wetted with saliva, vapor, and respiratory droplets, the silver and zinc generate a weak electric field that repels viruses on the surface. The use of
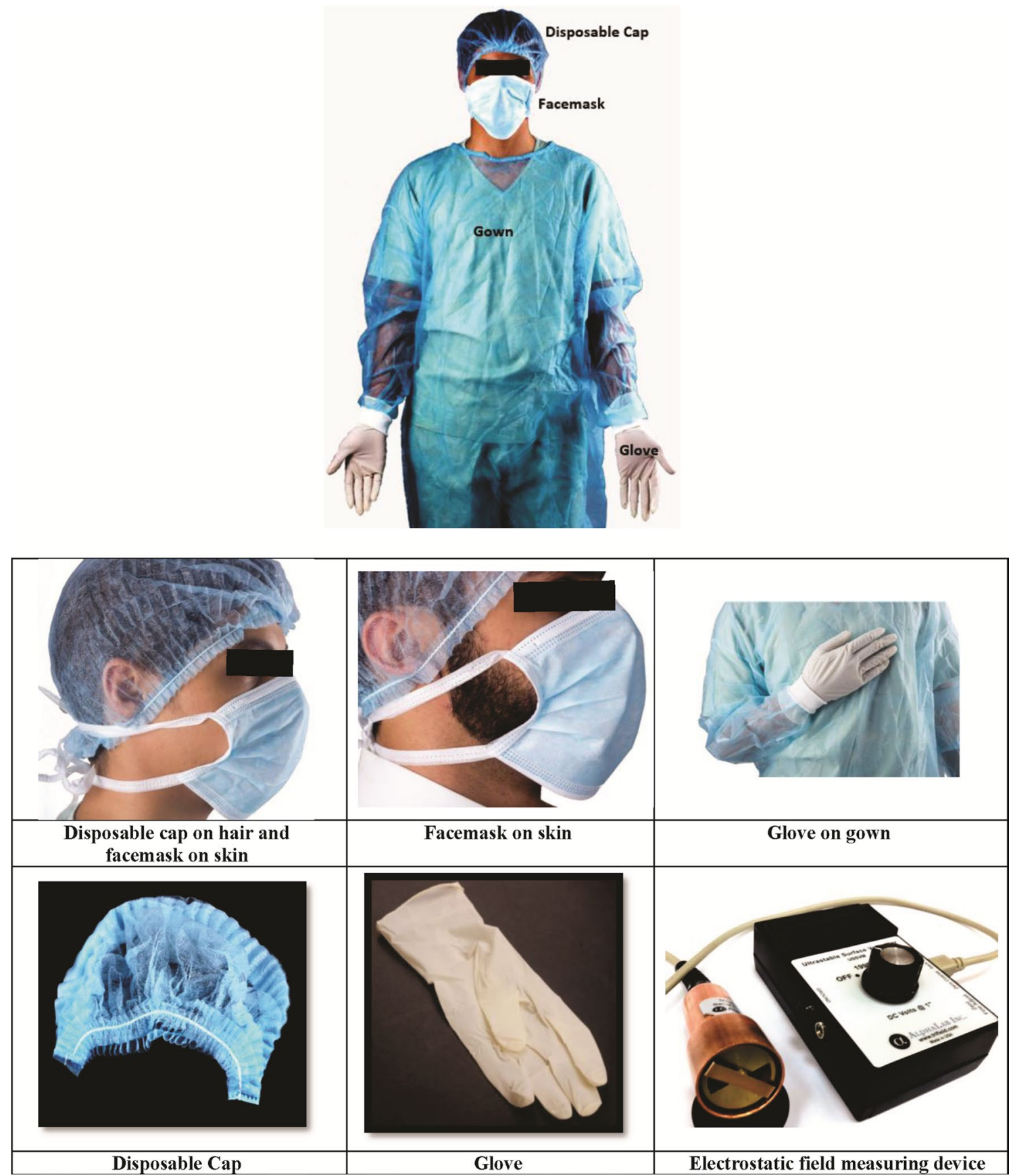

Fig. 1 The tested items, materials, and surfaces as well as the measuring device 
Fig. 2 Arrangement of the test procedures

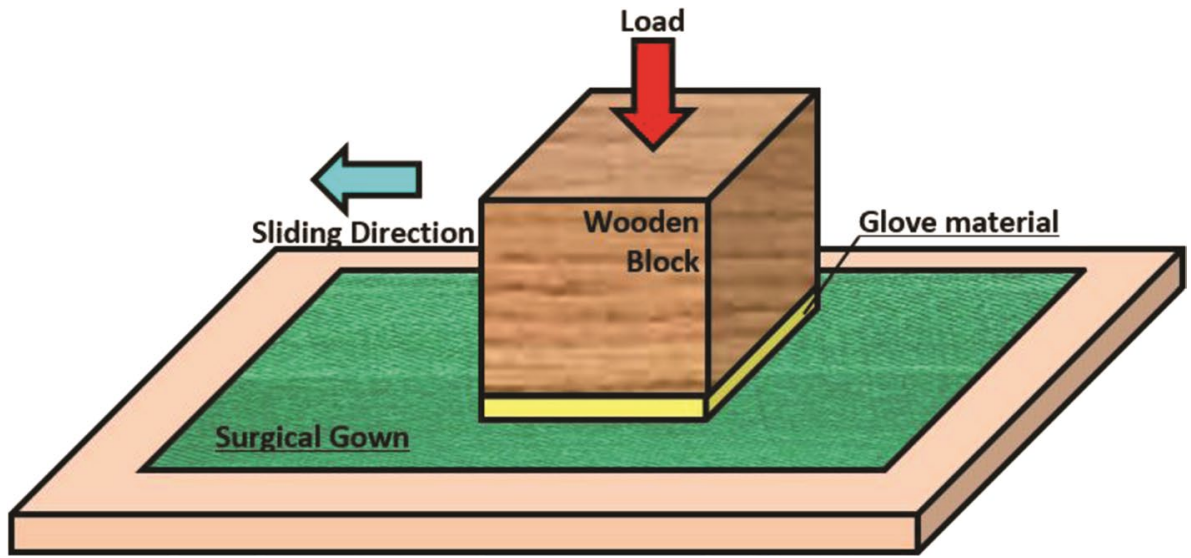

Fig. 3 (A) Generation of ESC after separation (B) Generation of ESC after sliding
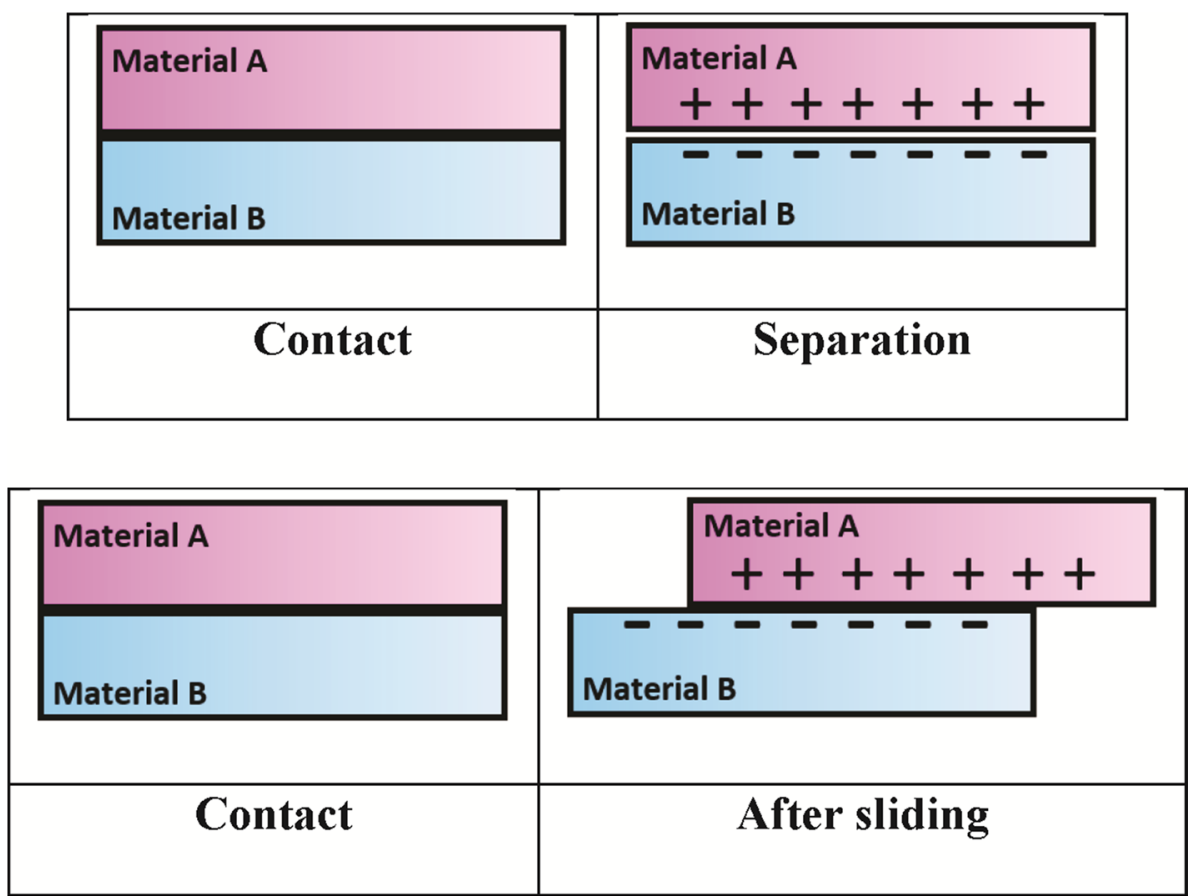

a combination of woven cotton and silk or polyester chiffon as the electrostatic layers [15] can increase the filtration efficiency.

Meanwhile, personal protective equipment (PPE) is made of polymeric materials that are easily triboelectrified; that is, they gain a positive or negative ESC from rubbing against each other. The resulting electric field can attract or repel electrically charged particles, including viruses [3]. The triboelectric series ranks materials according to their ESC gained by triboelectrification $[4,7,25]$. Recent studies have highlighted surgical masks as a good option for retarding the spread of COVID-19 due to its nature as a respiratory disease $[2,5,8,16,22,24]$. It was subsequently proposed to make use of an ESC generated on the surgical masks surface to capture or repel the viruses [1]. More broadly, to ensure that PPE is properly protective, it is necessary to know the sign and intensity of ESC gained by the polymeric material of which it is composed. Hence, in this study, we examined triboelectrification of polymeric materials used in manufacturing medical PPE and recommend specific materials that will enhance the wearer's safety.

\section{Materials and methods}

We investigated the ESCs generated by the contact, separation, and sliding of items such as facemasks, gloves, disposable caps, and gowns (Fig. 1). The facemasks and gowns were made of polypropylene (PP), the caps were made of polyethylene (PE), and the gloves were made of latex. Electrostatic fields on the tested items were measured in terms of voltage 


\begin{tabular}{|c|c|}
\hline Positive charge & \\
\hline Air & \\
\hline Silicone elastomer & $\overline{2} \bar{Z}$ \\
\hline Human hands & : 돌 \\
\hline Window glass & $\stackrel{2}{g}$ \\
\hline Rabbit fur & $\div 0$ \\
\hline Polymethyl methacrylate & 包 \\
\hline Human hair & 흘 \\
\hline Polyamide & $\sum^{5} \Sigma$ \\
\hline Aluminum & \\
\hline Paper & \\
\hline Cellulose acetate & \\
\hline Cotton & \\
\hline Polyurethane elastomer & \\
\hline Wood & \\
\hline Styrene-butadiene & $\mho_{2}$ \\
\hline Polystyrene & $\widehat{0}$ \\
\hline Hard rubber & 竞 \\
\hline Polyethylene glycol terephthalate & 氖 \\
\hline Epoxide resin & $\frac{1}{2} \sum^{2}$ \\
\hline Polyester & 象 \\
\hline Natural rubber & 包 \\
\hline Polyacrylonitrile & $\sum^{ \pm}$ \\
\hline Polystyrene & \\
\hline Polyethylene & \\
\hline Polypropylene & \\
\hline Polyvinyl chloride & \\
\hline Polytetrafluoroethylene & \\
\hline Negative charge & \\
\hline
\end{tabular}

Fig. 4 Triboelectric series of engineering materials

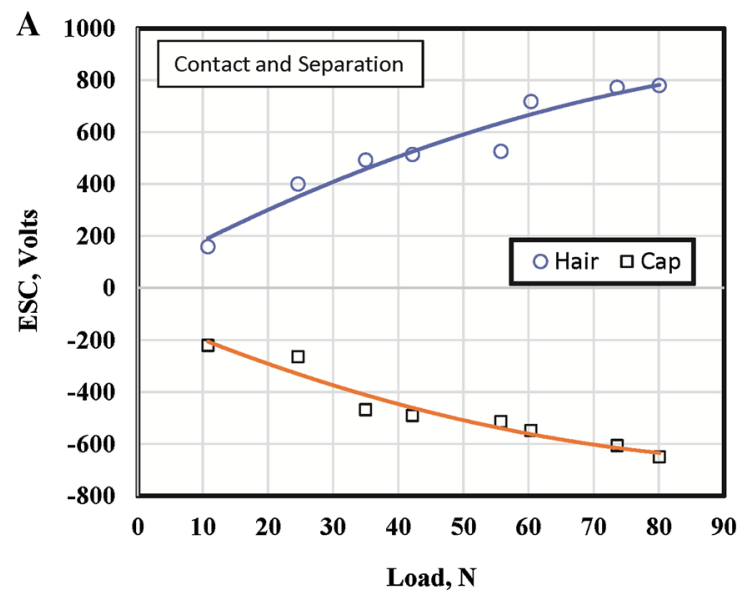

using an Ultra Stable Surface DC Voltmeter. Readings were recorded by a sensor $25 \mathrm{~mm}$ from the tested surface. The tested materials were attached to a wooden block with a surface of $50 \times 50 \mathrm{~mm}$. Specimens used as the counter-face were prepared in strips of $100 \mathrm{~mm}$ width and $300 \mathrm{~mm}$ length and fastened to a wooden plate with a surface of $400 \times 400$ mm (Fig. 2). Tests were carried out at room temperature under normal loads ranging from 10 to 80 newtons $(\mathrm{N})$. The wooden block was slid manually for a distance of $200 \mathrm{~mm}$. After contact and separation or sliding, the ESC was measured using the DC voltmeter.

\section{Results and discussion}

Contact and separation or sliding of dissimilar materials generates an ESC, during which each material gains charges of opposite polarity (Fig. 3). Polymeric materials are ranked according to their relative polarity in what is called a "triboelectric series". Materials that gain a positive charge when rubbed with another material are ranked more highly (Fig. 4). This ranking series allows the relative charge polarity and intensity of a set of materials to be determined.

The results of the experiment carried out to measure ESC from contact and separation along with sliding of the tested materials are presented in Figures 5, 6, 7, and 8. With contact and separation of the disposable cap and hair (Fig. 5A), the hair gained a positive charge and the cap gained a negative charge. Moreover, the generated ESC increased significantly when increasing the applied load, with the negative ESC ranging from -220 to -650 volts. Sliding of the disposable cap on hair generated still higher values of ESCs, reaching maximum values of 2846 and -3804 volts for hair and the disposable cap, respectively (Fig. 5B). It is well known that $\mathrm{PE}$, from which the disposable cap was made, develops a

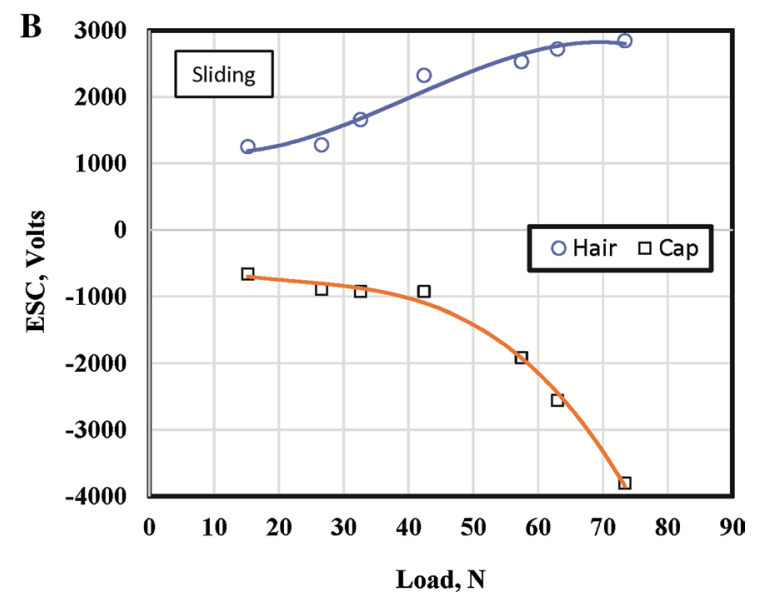

Fig. 5 (A) ESC generated from contact and separation of the disposable cap and hair (B) ESC generated from sliding of the disposable cap on hair 

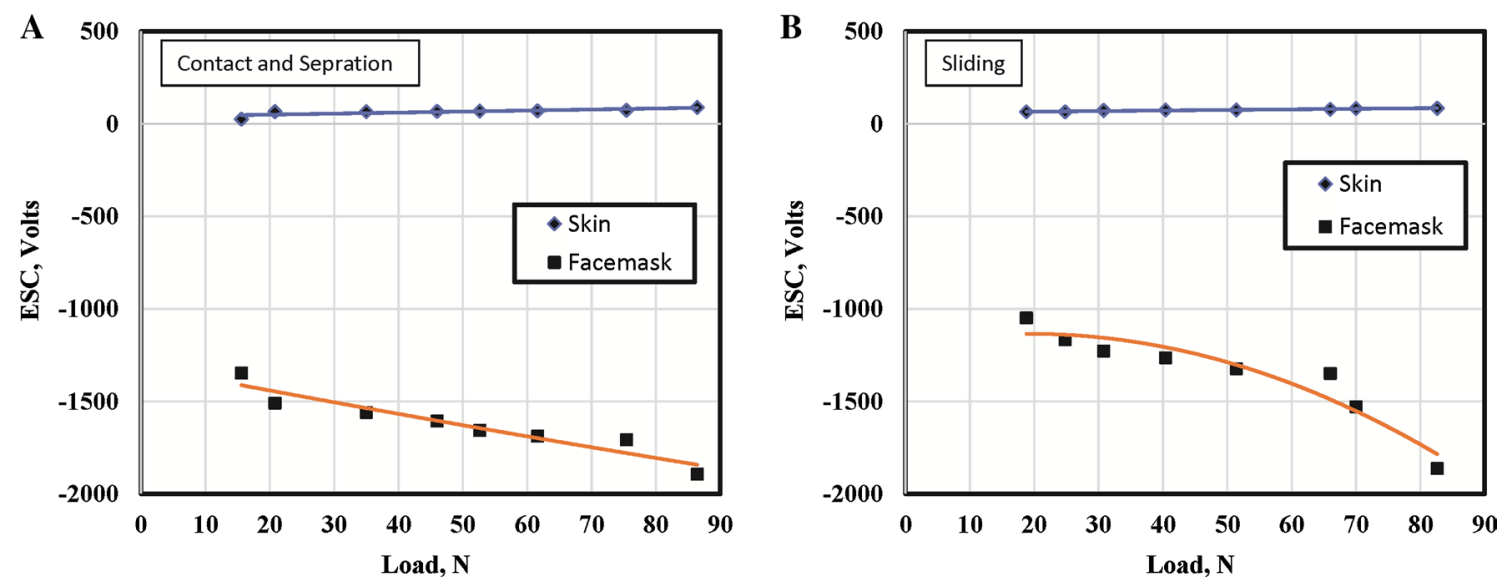

Fig. 6 (A) ESC generated from contact and separation of the facemask and skin (B) ESC generated from sliding of the facemask on skin
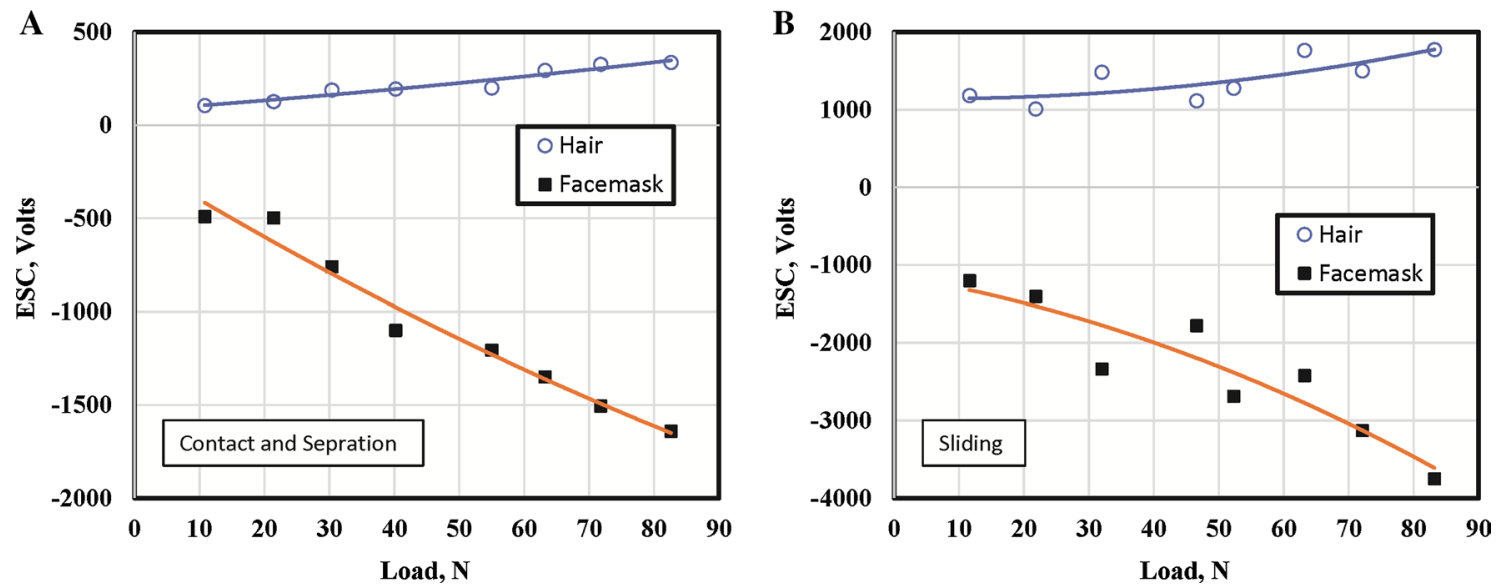

Fig. 7 (A) ESC generated from contact and separation of the facemask and hair (B) ESC generated from sliding of the facemask on hair
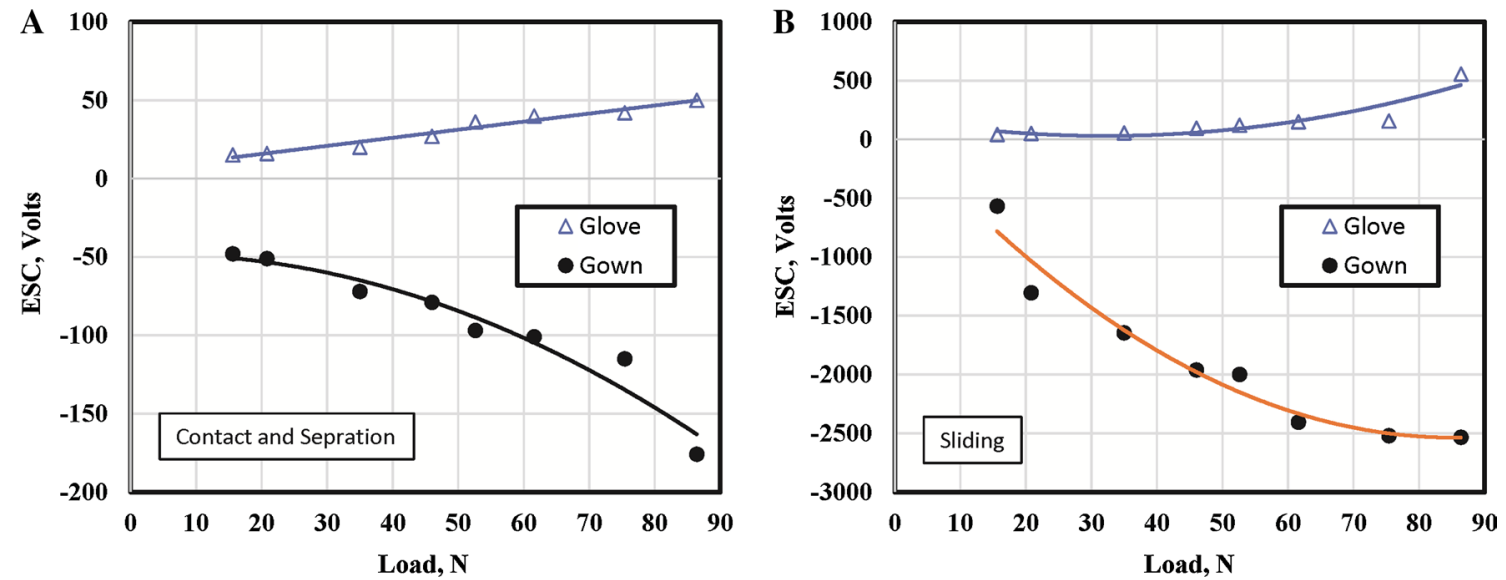

Fig. 8 (A) ESC generated from contact and separation of the glove and gown (B) ESC generated from sliding of the glove and gown 
negative charge when in contact with hair. The high negative ESC gained by the cap is expected to repel viruses, which also have negative ESCs, preventing them from adhering to the outer layer of the cap.

The generation of ESCs from contact and separation of facemask and skin is shown in Figure 6A. Values measured for the skin are very low due to the good electrical conductivity of the human body, while the facemask acquired higher negative values of up to -1984 volts. In terms of the safety of medical protective equipment, the choice of PP for the facemask is quite good because PP is ranked in the triboelectric series as an aggressively negatively charged material. This property guarantees the repulsion of viruses away from the facemask; indeed, it is known that generation of a negative ESC on the surface of a facemask can be considered a means of exerting a repulsive force against viruses in the air. The same trend in ESC values was observed for sliding of the facemask on skin (Fig. 6B).

ESC data from the contact and separation of facemask and hair are shown in Figure 7A. The facemask gained -1641 volts at an $82 \mathrm{~N}$ load, while under the sliding interaction, the ESC increased to -3751 volts (Fig. 7B), which is even higher than that measured for the contact and separation of facemask and skin. Generally, the values were high enough to repel negatively charged viruses. When using a positively charged material for a facemask or disposable cap, it cannot be guaranteed that the wearer will be protected; moreover, the external surfaces of the facemask and cap would themselves be sources of infection.

The ESC values generated from contact and separation of the gloves and gown were relatively low (Fig. 8A). The highest value gained by a glove was 50 volts, while the maximum gained by a gown was -176 volts. Sliding of a glove on a gown resulted in higher values (Fig. 8B), with the glove gaining 555 volts, while the gown achieved -2534 volts. The positive ESC generated on the glove surface is of great concern, because it can attract negatively charged viruses and enable infection of other individuals. Meanwhile, the gown can be considered safe due to its negative ESC. In a recent study, PE was recommended as a material for gloves and gowns in order to achieve lower ESC values.

\section{Summary and conclusions}

Contact and separation or sliding of a disposable cap and hair generated high ESC values. The disposable cap gained a negative charge, which is expected to repel viruses and prevent them from adhering to the outer layer of the cap. Likewise, the choice of PP as a material for facemasks is quite good. PP is ranked in the triboelectric series as an aggressively negatively charged material, which makes it likely to repel viruses. It is known that generation of a negative ESC on a facemask surface can be a means of exerting a repulsive force against viruses in the air. When testing facemasks, higher ESC values were achieved for contact and separation with hair than with skin. Finally, sliding of gloves on gowns achieved higher ESC values than did their contact and separation. However, the positive ESC generated on the glove surface is of great concern because it can attract negatively charged viruses and act as a source of infection. Manufacturing gloves and gowns from PE is recommended for ensuring lower ESC values.

Acknowledgements Taif University Researchers Supporting Project number (TURSP-2020/51), Taif University, Taif, Saudi Arabia. The sponsor had no role in study design; in the collection, analysis, and interpretation of data; in the writing of the report; or in the decision to submit the article for publication.

Author contributions All four authors made substantial contributions to all of the following: (1) the conception and design of the study, acquisition of data, analysis and interpretation of data; (2) drafting the article and revising it critically for important intellectual content; and (3) final approval of the final version.

Funding This research did not receive any specific grant from funding agencies in the public, commercial, or not-for-profit sectors.

Data Sharing and Data Accessibility All data pertinent to this article are included herein.

\section{Declarations}

Conflict of interest The author(s) declare that they have no conflict of interest.

Research involving human participants and/or animals Not applicable.

\section{References}

1. Ali AS, Ali WY (2020) Proper material selection of medical gloves. J Egypt Soc Tribol 17:1-11

2. Benson SM, Novak DA, Ogg MJ (2013) Proper use of surgical N95 respirators and surgical masks in the OR. AORN J 97:457-470

3. Brown R (1989) Effect of electric charge in filter materials. Filtration Separat 26:46-51

4. Burgo TA, Galembeck F, Pollack GH (2016) Where is water in the triboelectric series? J Electrostat 80:30-33

5. Chellamani K, Veerasubramanian D, Balaji RV (2013) Surgical face masks: manufacturing methods and classification. J Acad Ind Res 2:320-324

6. Crowell R (2020) Coronavirus on Masks and Clothing: New materials and coatings could make fabric inactivate or repel viral particles. Sci Am

7. Gooding DM, Kaufman GK (2011) Tribocharging and the triboelectric series. Encycl Inorg Bioinorg Chem 2011:1-14

8. Greenhalgh T, Schmid MB, Czypionka T, Bassler D, Gruer L (2020) Face masks for the public during the covid-19 crisis. BMJ 2020:369 
9. Hernando-Pérez M, Cartagena-Rivera A, Božič AL, Carrillo PJ, San Martín C, Mateu MG, Raman A, Podgornik R, De Pablo P (2015) Quantitative nanoscale electrostatics of viruses. Nanoscale 7:17289-17298

10. Hogan CJ, Lee M-H, Biswas P (2004) Capture of viral particles in soft X-ray-enhanced corona systems: charge distribution and transport characteristics. Aerosol Sci Technol 38:475-486

11. Janssen LL, Bidwell JO, Mullins HE, Nelson TJ (2003) Efficiency of degraded electret filters: part I. Laboratory testing against $\mathrm{NaCl}$ and DOP before and after exposure to workplace aerosols. J Int Soc Respir Prot 20:71-80

12. Janssen LL, Bidwell JO, Mullins HE, Nelson TJ (2003) Efficiency of degraded electret filters: Part II. Field testing against workplace aerosols. J Int Soc Respir Prot 20:81-90

13. Kashyap U, Saha SK (2020) Enhanced Design of PPE Based on Electrostatic Principle to Eliminate Viruses (SARS-CoV-2). Trans Indian Natl Acad Eng 2020:1

14. Kulkarni P, Namiki N, Otani Y, Biswas P (2002) Charging of particles in unipolar coronas irradiated by in-situ soft X-rays: enhancement of capture efficiency of ultrafine particles. J Aerosol Sci 33:1279-1296

15. Leung WWF, Sun Q (2020) Electrostatic charged nanofiber filter for filtering airborne novel coronavirus (COVID-19) and nanoaerosols. Separat Purif Technol 2020:116886

16. Lipp A, Edwards P (2002) Disposable surgical face masks for preventing surgical wound infection in clean surgery. Cochrane Datab Syst Rev 130:269-296

17. Liu C, Xie X, Zhao W, Liu N, Maraccini PA, Sassoubre LM, Boehm AB, Cui Y (2013) Conducting nanosponge electroporation for affordable and high-efficiency disinfection of bacteria and viruses in water. Nano Lett 13:4288-4293

18. Liu C, Xie X, Zhao W, Yao J, Kong D, Boehm AB, Cui Y (2014) Static electricity powered copper oxide nanowire microbicidal electroporation for water disinfection. Nano Lett 14:5603-5608
19. Lodish H, Berk A, Kaiser CA, Krieger M, Scott MP, Bretscher A, Ploegh H, Matsudaira P (2008) Molecular cell biology. Macmillan

20. Saikatendu KS, Joseph JS, Subramanian V, Neuman BW, Buchmeier MJ, Stevens RC, Kuhn P (2007) Ribonucleocapsid formation of severe acute respiratory syndrome coronavirus through molecular action of the $\mathrm{N}$-terminal domain of $\mathrm{N}$ protein. J Virol 81:3913-3921

21. Valentini L, Bittolo Bon S, Giorgi G (2020) Engineering graphene oxide/water interface from first principles to experiments for electrostatic protective composites. Polymers 12:1596

22. World-Health-Organization (2020) Modes of transmission of virus causing COVID-19: implications for IPC precaution recommendations: scientific brief, 27 March 2020. No. WHO/2019-nCoV/Sci Brief/Transmission_modes/2020.1. World Health Organization

23. Yao Q, Masters PS, Ye R (2013) Negatively charged residues in the endodomain are critical for specific assembly of spike protein into murine coronavirus. Virology 442:74-81

24. Zhang R, Li Y, Zhang AL, Wang Y, Molina MJ (2020) Identifying airborne transmission as the dominant route for the spread of COVID-19. Proc Natl Acad Sci 117:14857-14863

25. Zou H, Zhang Y, Guo L, Wang P, He X, Dai G, Zheng H, Chen C, Wang AC, Xu C (2019) Quantifying the triboelectric series. Nature Commun 10:1-9

Publisher's Note Springer Nature remains neutral with regard to jurisdictional claims in published maps and institutional affiliations. 